\title{
Acute and chronic evolution of MRI findings in a case of posterior spinal cord ischemia
}

\author{
This article has been corrected since Online Publication and a corrigendum has also been published
}

\author{
L Grassner ${ }^{1,2,3}$, F Klausner ${ }^{4}$, M Wagner ${ }^{5}, \mathrm{M} \mathrm{McCoy}^{4}$, S Golaszewski ${ }^{1}$, S Leis ${ }^{1}$, L Aigner ${ }^{2,6}$, \\ S Couillard-Despres ${ }^{2,3}$ and E Trinka ${ }^{1,2}$
}

Study design: Case report.

Objective: Reveal the evolution of the magnetic resonance imaging (MRI) pattern in a patient with a posterior spinal artery infarction, which belongs to a subgroup of spinal cord ischemia syndromes and presents a rare cause of spinal cord injury. Our report underlines that diagnosis of spinal cord ischemia and thus clinical decision making remains challenging.

Setting: University Hospital of Innsbruck and University Hospital of Salzburg, Austria.

Methods: Here we present clinical, electrophysiological and imaging data in the acute, subacute and chronic phase of a woman who developed signs and symptoms related to a bilateral posterior spinal cord infarction.

Results: At the clinical nadir (24 h after symptom onset), MRI did not exhibit T2 hyperintensities. However, such MRI changes were detected 8 days after symptom onset and persisted until the latest follow-up at 5 months.

Conclusions: Repeated MRI constitutes an indispensable diagnostic and follow-up tool for spinal cord ischemia. The imaging data in accordance with the electrophysiological measurements correlated well with the clinical presentation in the subacute und chronic phase. Therefore, further studies might allow using MRI following spinal cord ischemia as a prognostic marker for an individual outcome.

Spinal Cord (2014) 52, S23-S24; doi:10.1038/sc.2013.165; published online 14 January 2014

Keywords: non-traumatic spinal cord injury; posterior spinal artery syndrome; posterior spinal artery infarction; spinal cord diseases; MR imaging

\section{INTRODUCTION}

Spinal cord infarction accounts for a minority of all strokes. The small number of patients leads to limited experience with this potentially devastating pathology. Larger clinical trials do not exist. Therefore the clinical outcome is hard to predict.

Arterial infarcts to the posterior spinal artery present a peculiar rare incidence and pathology. The scarcity of this condition is most likely due to the extensive branching of the bilateral posterior spinal arteries. Moreover, the posterior column might be less susceptible to ischemia as it receives the lowest blood flow of all tracts in the spinal cord. ${ }^{1}$ Modern imaging techniques have the potential to assist the clinician in decision making regarding diagnosis and in the evaluation of potential therapeutic interventions.

\section{CASE REPORT}

Here we present a 79-year-old Caucasian woman with a history of arterial hypertension and moderate smoking, who developed a tingling sensation and weakness in both legs a few seconds after being frightened by a dog. Approximately $30 \mathrm{~min}$ later she noticed dropping of her right forefoot while she was ascending a staircase, a heat sensation on the lateral edge of her tibial bone and a numb feeling in her buttocks, but no clear sensory level. In addition, she realized problems with initiating and voiding her bladder. She was transferred to an emergency department, where an magnetic resonance image (MRI) showed a narrowing of the intervertebral disc space with a punctum maximum between Th12/L1, L3/L4 and L4/L5. The spinal cord did not show any abnormality at this time. Because of her clinical signs and the imaging findings, a discectomy at the level L4/L5 was performed.

The next morning she was unable to move her legs against gravity and presented with a hypoesthesia below the level L2, an anesthesia below L4, an absent anal reflex and a bilateral absent sensation of pain and vibration distally. T2-weighed MR images showed a faint increase in signal intensity in the posterior portion of the spinal cord from Th10 to Th12. In combination with the clinical time course and presentation, a bilateral infarct of the posterior spinal arteries was suspected. She was immediately started on intravenous heparin. After a period of 10 days, she received lowmolecular-weight heparin subcutaneously and a low dose of aspirin (100 mg/daily).

T2w MRIs 8 days after symptom onset showed a clear contrastenhancing dorsomedullary lesion from level Th10 to Th12.

\footnotetext{
${ }^{1}$ Department of Neurology, Christian Doppler Klinik, Paracelsus Medical University, Salzburg, Austria; ${ }^{2}$ SCI-TReCS (Spinal Cord Injury and Tissue Regeneration Center Salzburg), Paracelsus Medical University, Salzburg, Austria; ${ }^{3}$ nnstitute of Experimental Neuroregeneration, Paracelsus Medical University, Salzburg, Austria; ${ }^{4}$ Division for Neuroradiology, Christian Doppler Klinik, Paracelsus Medical University, Salzburg, Austria; ${ }^{5}$ Department of Neuroradiology, University Hospital Innsbruck, Innsbruck, Austria and ${ }^{6}$ Institute of Molecular Regenerative Medicine, Paracelsus Medical University, Salzburg, Austria

Correspondence: Professor E Trinka, Department of Neurology, Christian Doppler Klinik, Paracelsus Medical University, Ignaz Harrer Straße 79, Salzburg 5020, Austria.

E-mail: E.Trinka@salk.at
}

Received 24 August 2013; revised 4 December 2013; accepted 11 December 2013; published online 14 January 2014 

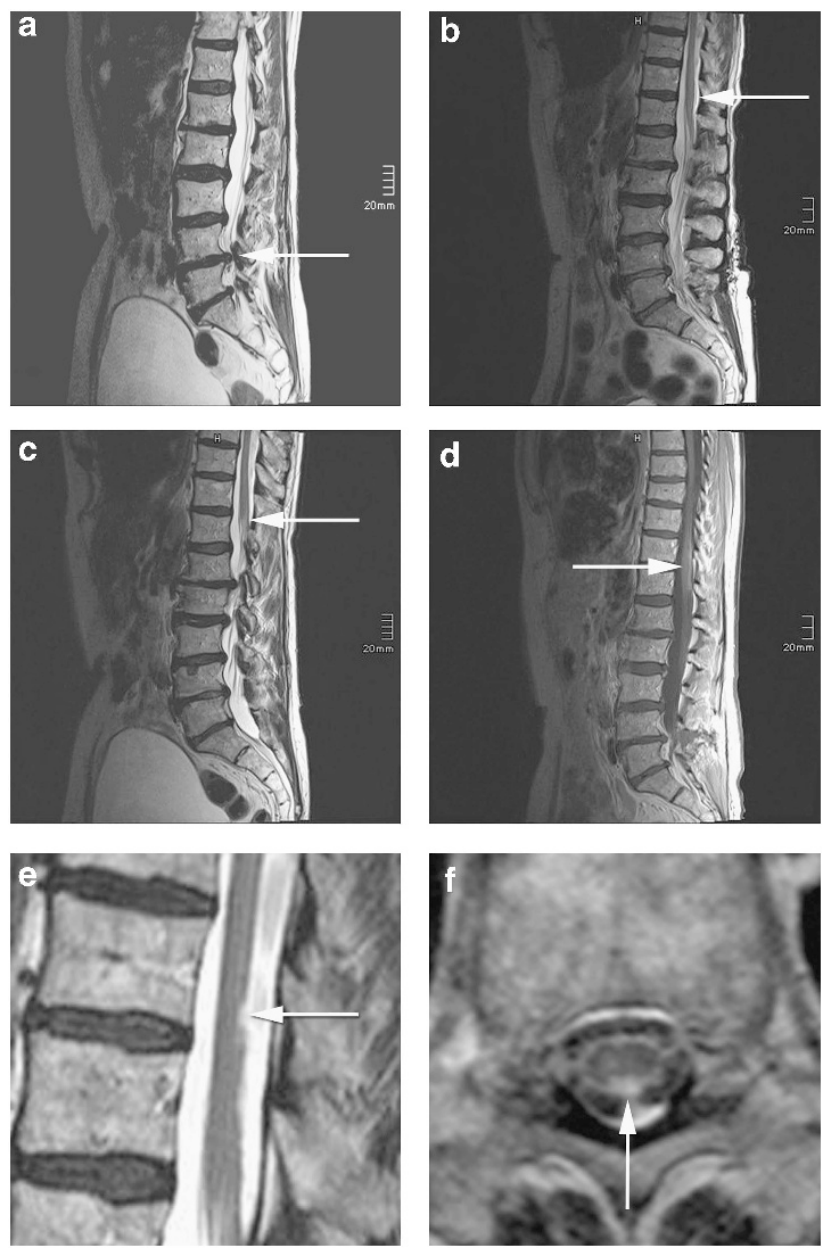

Figure 1 (a) Sagittal T2w MR image showing a lateral L4/L5 disc herniation towards the right (b) Sagittal T2w MRI 8 h post onset of symptoms with no signal alteration within the spinal cord. (c) Sagittal T2w MRI 1 day-post onset of symptoms with slight intramedullary signal increase at level Th10/11. (d) Sagittal T1w MRI 8 days post onset of symptoms showing demarcation of circumscribed dorsomedullary signal increase at level Th10/ 11 till Th12. (e) Sagittal T2w MRI 5 months post onset with MRI showed a small (about $3 \mathrm{~mm}$ ) T2 hyperintense lesion with low signal on T1 in the region of the posterior columns compatible with a residual gliotic scar at the level of Th10. (f) Corresponding axial T2w MRI to (e) showing a clear demarcation of a hyperintense lesion in the dorsal columns bilaterally at level of Th10 as an imaging correlate of cystic transformation of the ischemic lesion.

Somatosensory-evoked potentials showed no cortical response upon stimulation on either side and transcranial motor-evoked potentials were not elicitable. An extensive diagnostic work-up revealed no definite etiologic cause for the infarction. Three weeks after the onset, the patient was sent to an early neurological rehabilitation unit with some residual motor strength. She slowly improved under extensive rehabilitation measurements.

After 5 months, she was able to ambulate with a wheeled walker by herself, was able to stand alone without help and regained some proprioceptive function. Her bladder function fully recovered. An MRI showed in T2w, in sagittal and axial orientation, a clear demarcation of a cystic lesion at level T11 as residuum of an intramedullar ischemia. Somatosensory potentials were still absent from her lower extremities, but motor-evoked potentials were elicitable in both the legs (Figure 1).

\section{DISCUSSION}

Our case highlights the diagnostic and therapeutic dilemma of spinal cord ischemia. Spinal cord infarction does not necessarily have the same risk group compared with its cerebral counterpart. ${ }^{2}$ Mechanical triggering movements and prior diseases of the spine seem to be risk factors. ${ }^{3}$ However, there is no single pathognomonic marker with a clear prognostic value. Modern imaging modalities might represent an excellent candidate to make an accurate diagnosis and to evaluate any improvement or deterioration.

Previous studies on patients with spinal cord infarction suggest a restricted diffusion on diffusion-weighted images during the early phase, ${ }^{4}$ T2 signal abnormalities and the diagnostic value of MRI in combination with electrophysiological examinations. ${ }^{5}$ Our patient however showed no T2 hyperintensity after $24 \mathrm{~h}$, but after 8 days. One could speculate that this condition might have been visible earlier with a higher resolution. T2 hyperintensities were still present and, interestingly, DWI values were still reduced after 5 months. This case illustrates the potential necessity of serial imaging with MRI if spinal cord ischemia is suspected, as initial scans may not appear abnormal. Importantly, the imaging findings correlated well with the clinical and electrophysiological examinations during the observed period.

\section{CONCLUSION}

In summary, we present a woman with bilateral posterior spinal artery infarction. Despite the initial severity, she improved constantly throughout her hospital stay and neurorehabilitation seemed to be justified even months after. Related to the paucity of posterior spinal cord syndromes, there is no experience with the typical time course and imaging pattern of this pathological condition, indicating the need for further investigation and larger studies.

\section{CONFLICT OF INTEREST}

The authors declare no conflict of interest.

1 Blisard KS, Follis F, Wong R, Miller KB, Wernly JA, Scremin OU. Degeneration of axons in the corticospinal tract secondary to spinal cord ischemia in rats. Paraplegia 1995, 33: $136-140$.

2 Naess H, Romi F. Comparing patients with spinal cord infarction and cerebral infarction: clinical characteristics, and short-term outcome. Vasc Health Risk Manag 2011; 7: 497-502.

3 Novy J, Carruzzo A, Maeder P, Bogousslavsky J. Spinal cord ischemia: clinical and imaging patterns, pathogenesis, and outcomes in 27 patients. Arch Neurol 2006; 63: 1113-1120.

4 Thurnher MM, Bammer R. Diffusion-weighted MR imaging (DWI) in spinal cord ischemia. Neuroradiology 2006; 48: 795-801.

5 Nardone R, Bergmann J, Kronbichler M, Lochner P, Caleri F, Tezzon F et al. Magnetic resonance imaging and motor-evoked potentials in spinal cord infarction: report of two cases. Neurol Sci 2010; 31: 505-509. 